\title{
Epidemiology and patterns of hospital use after parasuicide in the south west of England
}

\author{
David J Gunnell, Jane Brooks, Tim J Peters
}

\begin{abstract}
Objective - To describe the epidemiology,

patients who do not require admission on medical grounds.
\end{abstract} management, and outcome of parasuicide in the south west of England.

Design - Descriptive analysis using routine information systems.

Setting - The former South Western Regional Health Authority, population aged 10 and over: 2.9 million.

Subjects - These comprised 5080 residents of the South Western Health Region, admitted on 5770 occasions for parasuicide between April 1992 and March 1993 to hospitals in the south west.

Main outcome measures - Person based age and sex standardised admission ratios for parasuicide; readmission for parasuicide in the subsequent 12 months; admission to psychiatric hospital after parasuicide; in-hospital mortality for those admitted after attempted suicide.

Results - The overall rate of parasuicide admission was 174 per 100000 per year. Rates were highest in males aged 20-24 (381 per 100000 ) and in females aged 15-19 (625 per 100000 ). Parasuicide is the third most frequent cause of acute medical admission in the south west. A total of $10.0 \%$ of admissions received psychiatric inpatient care as a direct result of their parasuicide admission and $10 \cdot 1 \%$ were readmitted in the following 12 months with a repeat episode of parasuicide. Significant variations in standardised admission ratios for parasuicide were observed between the districts. Some of this variation is related to socioeconomic differences between districts, the rest is probably due to differences in practice between districts. There is no clear evidence that these variations result in differences in readmission rates. Districts where psychiatric inpatient facilities were located on the same site as the general hospital tended to admit a greater percentage of parasuicide patients for psychiatric inpatient care. A quarter of all suicide deaths from overdose occurred in hospital. It is estimated that there are 87000 parasuicide admissions in England and Wales annually.

Conclusions - Parasuicide is a common cause of acute hospital admission and there is evidence that hospital admission practices for parasuicide vary across the south west. Randomised controlled trials are needed to evaluate the most appropriate form of management for those (f Epidemiol Community Health 1996;50:24-29)

After showing a reduction in the 1980 s, parasuicide rates in this country are once again rising in some areas. ${ }^{1-4}$ Reduction in rates of both suicide and parasuicide have been identified as targets in health strategy documents in this country and elsewhere..$^{5-7}$ Both suicide and parasuicide are used in these documents as proxy indicators of the mental health of the population. While there is uncertainty about the value of suicide as an outcome indicator for mental illness services, parasuicide undoubtedly remains a common and important health care problem. Although some interventions for parasuicide are effective in reducing short term repetition rates in high risk groups, no randomised controlled trials have been large enough to show the effectiveness of interventions in unselected patient groups. ${ }^{8}$

In 1984, a Department of Health working group reversed previous recommendations that all deliberate self harm patients should be assessed by psychiatrists. ${ }^{9}$ The group acknowledged that some patients would be managed by their general practitioner alone and that others would not be admitted after attendance at accident and emergency departments. In this context there is evidence in recent years that admission practices vary between hospitals. In some hospitals less than half of those who attend accident and emergency after parasuicide are admitted, in others over $80 \%$ of attenders are admitted. ${ }^{2810} \mathrm{We}$ have examined the epidemiology of parasuicide in the south west region between April 1992 and March 1993 using routinely available data on hospital admissions. In particular, interdistrict variations in parasuicide admission rates and repetition rates are examined together with differences in management.

\section{Methods}

DATA SOURCES

Details on each hospital admission for parasuicide were obtained from routine health inWestern Health Region (Patient Administration System (PAS)). This records patient details (date of birth, sex, and postcode) and details of outcome (length of stay, death, discharge to another hospital) for all hospital admissions. Information on the methods used in formation systems used in the former South 

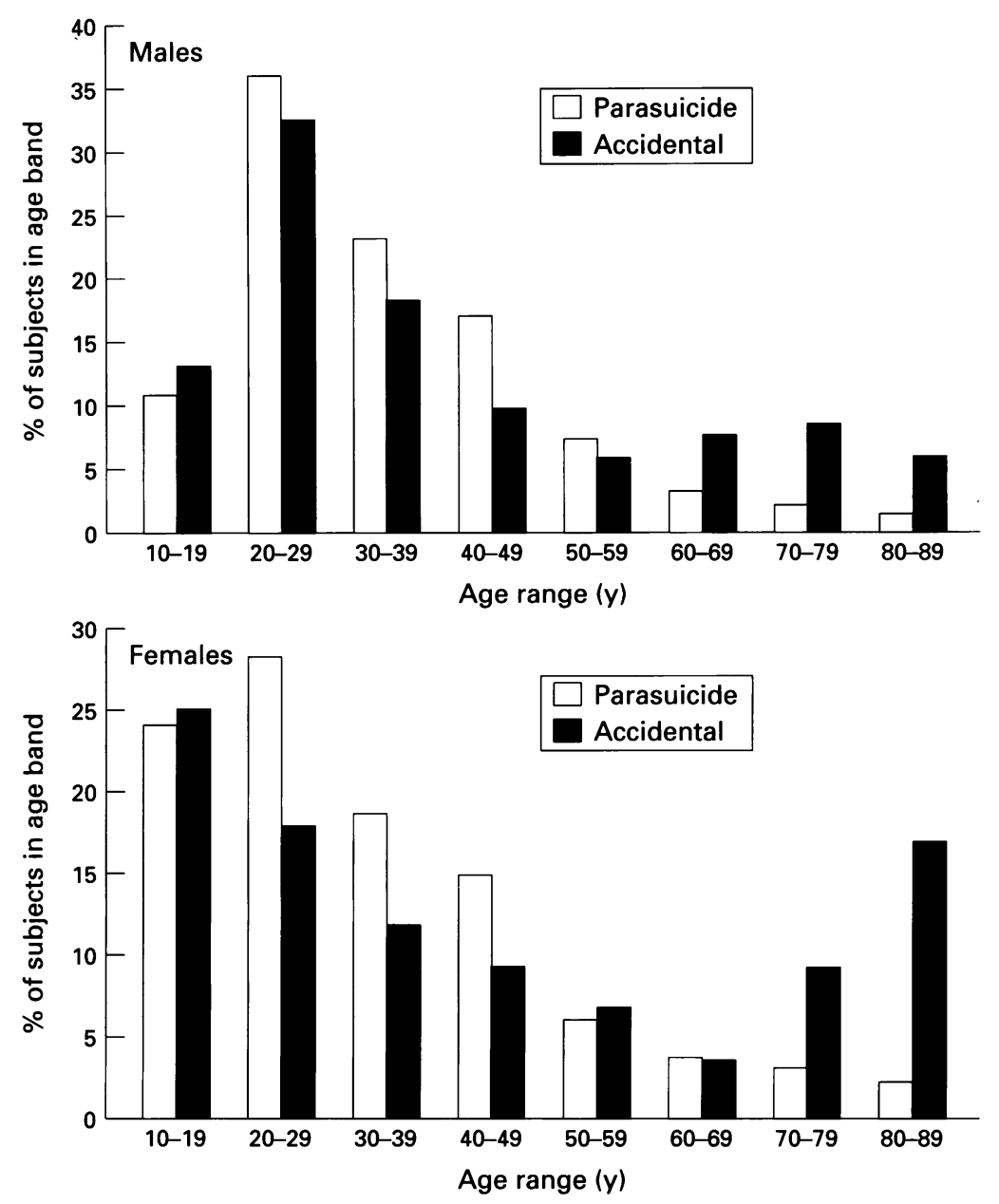

Figure 1 Comparison between parasuicide and accidental overdose admissions in males and females. poisoning is more likely. Individuals aged 10 years or less were omitted from all analyses as intentional self harm in children is rare, and is most likely to be accidental. No attempt was made to include accidental injuries in the same way as accidental poisoning. This decision was based on the assumption that wrist laceration coded as accidental is more likely to be truly accidental than is overdose coded as accidental, and inclusion of the former episodes may therefore distort the estimate of incidence. While there is no direct evidence for this assumption, ICD categories for accidental injury and poisoning are less specific for wrist laceration.

Population data were obtained from the 1991 census and OPCS mid year population estimates. Information on suicides and methods used for suicide in the south west was obtained from OPCS mortality files.

A postal questionnaire was sent to the 14 accident and emergency departments attached to general hospitals in the south west. Information was sought on the total number of annual attendances following drug overdose and the number of such attenders who were admitted.

\section{PERSON BASED ADMISSION AND READMISSION} RATES

To assess person based admission rates and assess the repeat parasuicides rates, separate episodes of care were linked using district number, date of birth, and sex. Each patient was tracked for a 12 month period after their first date of admission, and readmission following further episodes of parasuicide recorded. Residents receiving treatment outside the region (less than $2.5 \%$ of the total in those districts on the borders of the region) were omitted as were residents from other regions admitted to hospitals in the south west. In-hospital deaths subsequent to parasuicide admission were counted as suicides. chotropic agents). Each main health care provider within the region has a PAS module, thus ensuring $100 \%$ coverage of all local inpatient episodes for each of the eight health districts. All medical coders within the region are centrally trained and frequently audited. Senior coders meet every three months to discuss coding issues, thus increasing consistency between hospitals in the region.

The codes corresponding to parasuicide are ICD E950-E959 and E980-E989. However, some non-accidental episodes of drug poisoning may be coded as accidental (E850-9) and therefore to gain the most complete picture of parasuicide we combined both these categories in our analyses. In fact, in the 12 months examined, 375 admissions (179 male; 196 female) were coded as accidental poisoning in people aged over 10 years. Similarities in the age and sex distribution of those coded as accidental (fig 1) with "true" parasuicides suggest that deliberate self poisoning is sometimes miscoded. Inclusion of some "true" episodes of accidental poisoning in our data may therefore lead to slight over estimation of parasuicide rates (by a maximum $7 \%$ ). This is particularly so in the older groups where accidental self

\section{PSYCHIATRIC ADMISSIONS}

Inspection of the data suggested that in a number of units, particularly those with on-site psychiatric facilities, some parasuicide admissions are coded as both medical and psychiatric episodes of care. Similarly, the psychiatric assessment of patients on the medical wards after parasuicide may have been coded as separate consultant episodes of care. To overcome the resulting overestimation of psychiatric admission rates we calculated the rates using two bases. The first estimate includes all parasuicides who had a psychiatric finished consultant episode (FCE) during their period of inpatient admission. The second excluded all those patients whose length of psychiatric admission was less than two days.

\section{STATISTICAL ANALYSES}

Age and sex standardised admission ratios (SAR) for each district health authority were calculated by the indirect method of standardisation using OPCS mid-year 1992 regional 


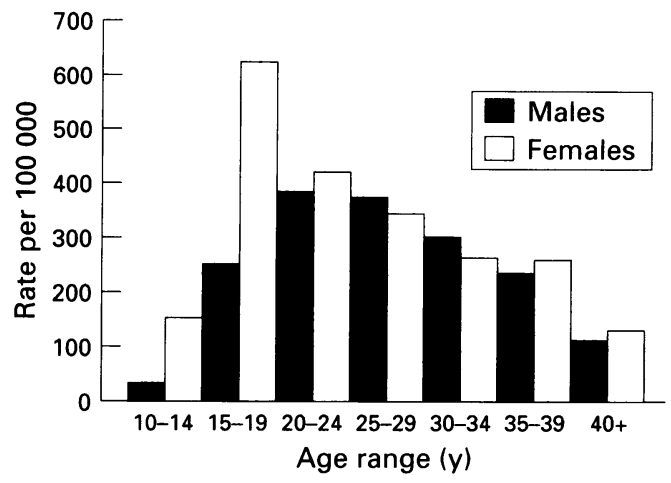

Figure 2 Parasuicide admission rates for different age groups in the South Western Health Region between April 1992 and March 1993.

population estimates. An estimate of the number of parasuicide admissions in England and Wales was obtained from the South Western Health Region age/sex specific rates multiplied by the England and Wales population for each age/sex population strata. Townsend scores were calculated for each of the districts from the 1991 census. ${ }^{11}$ These scores were used to examine the relationship between deprivation and SAR for parasuicide, and also to consider variations in admission rates both before and after adjustment for deprivation.

Standardisation indicates whether a given district's parasuicide experience is comparable to that of the regional population. This does not, however, give sufficient evidence of a differential pattern of admissions across districts; for this a comparative analysis of district parasuicide ratios is required. Assuming a Poisson distribution, it is possible to use an approximate $\chi^{2}$ test statistic to make a suitable comparison $^{33}$ since the standard employed here is the region itself.

\section{Results}

OVERALL RATES

Between April 1992 and March 1993 there were 5770 parasuicide admissions in the south west (admission rate for parasuicide 174 per 100000 per year). Altogether 5415 (93.8\%) admissions were for self poisoning and 2763 of these were following overdoses using analgesics, antipyretics, and anti-rheumatics (mainly paracetamol and aspirin). Parasuicide was the third commonest cause of acute medical admission after acute myocardial infarction

Table 1 Total parasuicide admissions in the south west April 1992-March 1993. Rates are indirectly standardised against the population of the former South Western Health

Region

\begin{tabular}{lrcl}
\hline District of residence & $\begin{array}{l}\text { District } \\
\text { population }\end{array}$ & $\begin{array}{l}\text { Total parasuicide } \\
\text { admissions }\end{array}$ & $\begin{array}{l}\text { Person based age/sex standardised } \\
\text { parasuicide admission ratio }(95 \% \\
\text { CI) }\end{array}$ \\
\hline 1 & 731289 & 1615 & $105 \cdot 8(99 \cdot 3,112 \cdot 5)$ \\
2 & 418197 & 840 & $107 \cdot 4(99 \cdot 7,115 \cdot 8)$ \\
3 & 283950 & 490 & $89 \cdot 8(81 \cdot 8,98 \cdot 6)$ \\
4 & 122722 & 310 & $130 \cdot 6(115 \cdot 2,148 \cdot 1)$ \\
5 & 294872 & 778 & $122 \cdot 4(113 \cdot 6,132 \cdot 0)$ \\
6 & 220476 & 312 & $80 \cdot 1(71 \cdot 0,89 \cdot 2)$ \\
7 & 472666 & 881 & $93 \cdot 0(86 \cdot 7,99 \cdot 7)$ \\
8 & 366587 & 544 & $78 \cdot 2(71 \cdot 5,85 \cdot 4)$ \\
South Western RHA & 2910759 & 5770 & 100 \\
\hline
\end{tabular}

(8685 admissions) and heart failure (6257 admissions).

A total of $2603(45 \cdot 1 \%)$ of the admissions involved males; 3167 (54.9\%) were female. The highest parasuicide rates in males were in those aged $20-24$ years (381 per 100000 ) and in woman rates were highest in those aged $15-19$ years (625 per 100000 ) (fig 2). Reasons for admission other than drug overdose included: injuries by cutting and piercing instruments (135), self inflicted poisoning by gases (58), injuries by jumping from high places (28), and the remainder (134) were admitted following attempted suicide by drowning, hanging, shooting, or unspecified means. Extrapolating these rates onto the population of England and Wales, it is estimated that there are in the order of 78500 admissions after episodes of deliberate self poisoning annually, and a total of 87000 admissions after deliberate self harm of some kind. Of these 39900 are for self poisoning with analgesics, antipyretics, and anti-rheumatics (mainly paracetamol and aspirin).

The total number of individuals admitted was 5080. Table 1 and figure 3 show the total number of parasuicide admissions and person based age and sex standardised admission ratios for each of the health districts. The standardised admission ratio for parasuicide ranged from 78.2 to 130.6 across the district health authorities. This variance was highly significant based on an approximate $\chi^{2}$ test for homogeneity $\left(\chi^{2}=107 \cdot 6\right.$ on $\left.7 \mathrm{df}, \mathrm{p}<0.001\right)$. Those districts with greater socioeconomic deprivation, as measured by the Townsend score, tended to have increased SARs for parasuicide (Spearman's rank correlation coefficient 0.31 $95 \%$ CI $-0.43,0.86$, fig 4 ). Using the ranked data, about $9 \%$ of the variance in parasuicide SAR was due to variation in Townsend score.

\section{MANAGEMENT}

The postal questionnaire was returned by 11 of the 14 casualty departments attached to general hospitals in the South Western Health Region. Two of the three departments that did not respond were within districts with other casualty departments from which responses were obtained. The sociodemographic mix of the populations served by the non-responding departments is typical of that in the south west. Reported admission rates after attendance at

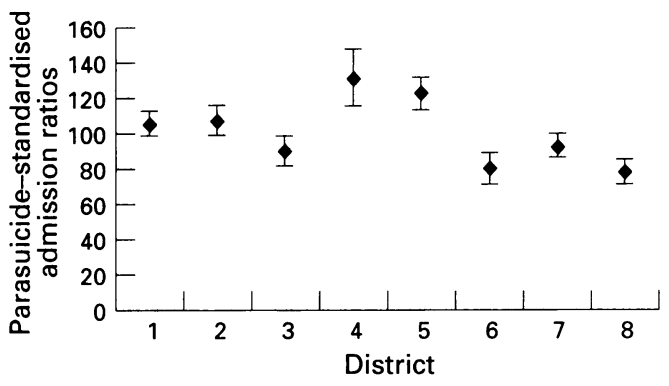

Figure 3 Person based standardised admission ratios for parasuicide in health districts in the South Western Health Region. 


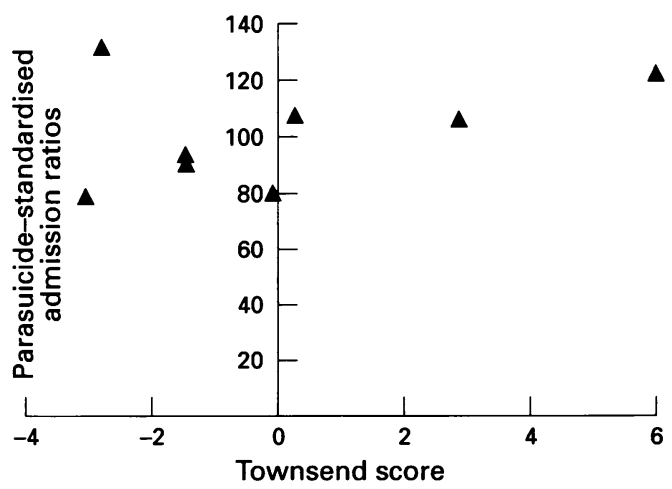

Figure 4 Relationship between the standardised admission ratio for parasuicide and the Townsend score for eight health districts in the South Western Health Region.

Table 2 Results of postal questionnaire to accident and emergency departments $(A \mathcal{E} E)$ in the south west regarding attendances and admission for parasuicide

\begin{tabular}{|c|c|}
\hline Location of $A \mathcal{E} E$ & Admission/AE $E$ attendances (\%) \\
\hline $\begin{array}{l}\text { District } 1 \\
\text { District } 1 \\
\text { District } 1 \\
\text { District 1 } \\
\text { District } 2 \\
\text { District } 4 \\
\text { District } 5 \\
\text { District } 6 \\
\text { District } 7 \\
\text { District } 7 \\
\text { District } 8\end{array}$ & $\begin{array}{l}712 / 991(71 \cdot 8) \\
259 / 436(59 \cdot 4) \\
228 / 295(77 \cdot 3) \\
226 / 334(67 \cdot 6) \\
245 / 554(44 \cdot 2) \\
104 / 128(81 \cdot 3) \\
742 / 974(76 \cdot 2) \\
231 / 447(48 \cdot 7) \\
422 / 636(66 \cdot 4) \\
110 / 185(59 \cdot 5) \\
300 / 418(71 \cdot 8)\end{array}$ \\
\hline
\end{tabular}

accident and emergency for parasuicide ranged from $44 \cdot 2 \%(95 \%$ CI $40 \cdot 1,48 \cdot 4 \%)$ to $81 \cdot 3 \%$ (95\% CI $74.5,88.0 \%$ ) (table 2). The health district with the highest SAR for parasuicide also reported the largest proportion of patients admitted after accident and emergency attendance $(81 \cdot 3 \%)$; Spearman's rank correlation coefficient between SAR for parasuicide and the percentage of accident and emergency attenders who were admitted was 0.43 (95\% CI $-0.48,0.89)$. The partial correlation coefficient (adjusted for Townsend score) was 0.55 (95\% CI $-0.35,0.92$ ).

The median length of inpatient stay was one day. This did not vary across the districts. Altogether $666(11.5 \%)$ patients were discharged to a psychiatric inpatient unit (table 3). If those patients whose psychiatric episode lasted less than two days are excluded, 579 $(10 \%)$ of parasuicide patients received subsequent inpatient psychiatric care. Districts where psychiatric inpatient facilities were located on the same site as general hospitals tended to admit a greater percentage of parasuicide patients for psychiatric inpatient care. There were significant variations between districts using either definition of psychiatric inpatient episode. There was a positive relationship between the parasuicide SAR and the proportion of patients admitted to psychiatric units (Spearman's $0.48 ; 95 \%$ CI $-034,0.89$; partial correlation coefficient, adjusted for Townsend: $0 \cdot 46$ ). For the seven districts where information on the percentage of patients admitted after parasuicide was obtained, there was no strong relationship (Spearman's 0.11; 95\% CI - 0.70, 0.80 ) between the percentage of individuals admitted and the number of subsequently referred for psychiatric care. Adjustment for Townsend score did not affect this.

\section{OUTCOME}

Altogether $512(10 \cdot 1 \%)$ patients were readmitted with one or more further episodes of parasuicide in the 12 months subsequent to their index admission. No relationship was found between SARs for parasuicide and the proportion of patients readmitted after parasuicide in the next 12 months (Spearman's rank correlation coefficient $0.07 ; 95 \%$ CI $-0.67,0.74$; partial correlation coefficient adjusted for Townsend: $0 \cdot 15$ ). There was a positive though non-significant relationship between the proportion of patients discharged to psychiatric hospitals and the proportion of patients readmitted with a diagnosis of parasuicide in the subsequent 12 months (Spearman's rank correlation coefficient: $0.55 ; 95 \%$ CI $-0.25,0.89$; partial correlation coefficient, adjusted for deprivation: $0 \cdot 52$ ).

Overall there were 42 in-hospital deaths ( $0.8 \%$ of all admissions) after parasuicide admission. Twenty nine (69\%) of these were after overdose.

\section{Discussion}

Hospital admission statistics underestimate total parasuicide episodes as many of those seen in casualty are not admitted to hospital. In addition, many cases may be managed in the community without the use of hospital services. $^{810^{12-1431}}$ Our aim, however, was to assess differences in hospital admission rates and the patterns of care among hospital admitted parasuicides. Our data are based on district of residence - we were not able to examine

Table 3 Proportion of patients undergoing further psychiatric inpatient care following parasuicide admission, and proportion of patients readmitted in the subsequent 12 months

\begin{tabular}{llll}
\hline District of residence & $\begin{array}{l}\text { No (\%) of parasuicide } \\
\text { admissions discharged to } \\
\text { psychiatric inpatient units* }\end{array}$ & $\begin{array}{l}\text { No (\%) of parasuicide admissions } \\
\text { where length of psychiatric inpatient } \\
\text { episode two days or more† }\end{array}$ & $\begin{array}{l}\text { No of patients (\%) readmitted } \\
\text { with parasuicide diagnosis in } \\
\text { the subsequent 12 mth }\end{array}$ \\
\hline 1 & $144(8 \cdot 9)$ & $129(8 \cdot 0)$ & $94(6 \cdot 6)$ \\
2 & $128(15 \cdot 2)$ & $111(13 \cdot 2)$ & $79(10 \cdot 5)$ \\
3 & $64(13 \cdot 1)$ & $55(11 \cdot 2)$ & $62(14 \cdot 3)$ \\
$4^{*}$ & $61(19 \cdot 7)$ & $49(15 \cdot 8)$ & $28(10 \cdot 4)$ \\
$5^{*}$ & $119(15 \cdot 3)$ & $102(13 \cdot 1)$ & $95(14 \cdot 3)$ \\
$6^{*}$ & $53(17 \cdot 0)$ & $44(14 \cdot 1)$ & $38(13 \cdot 3)$ \\
7 & $54(6 \cdot 1)$ & $52(5 \cdot 9)$ & $70(9 \cdot 0)$ \\
8 & $43(7 \cdot 9)$ & $37(6 \cdot 8)$ & $46(9 \cdot 4)$ \\
South Western RHA & $666(11 \cdot 5)$ & $579(10 \cdot 0)$ & $512(10 \cdot 1)$ \\
\hline
\end{tabular}

* Districts where psychiatric inpatient beds available on-site in the general hospitals $+\chi^{2}=95.5(p<0.001) ; \ddagger \chi^{2}=65.9(p<0.001)$. 
admission rates for individual hospitals (as denominator population data are not available). Differences between hospitals are likely to have been diluted in this aggregate level of analysis.

The reliability of hospital admission data on self poisoning in adolescents has been assessed in Oxford. ${ }^{32}$ Here over $95 \%$ of the episodes were correctly recorded. In the south west coders meet regularly to discuss coding issues and accuracy will probably have increased after the recent health service reforms with purchasers and providers placing increased importance on accurate recording of all episodes of hospital admission. To ensure complete case ascertainment, and minimise variations resulting from differences in coding, we examined all admissions whether coded accidental ( $7 \%$ of total), uncertain, or deliberate.

Parasuicide is the third commonest reason for acute medical admission in the south west. Over $90 \%$ of these admissions follow drug overdose, and of these over half involve drugs easily purchased over the counter in chemists and other retail outlets (paracetamol and aspirin). Admission rates were highest among men aged 20-24 and in women aged 15-19; similar to the pattern and rates found in $\mathrm{Ox}$ ford. ${ }^{2}$ The 12 month repeat rate of $10 \cdot 1 \%$ is slightly lower than that reported in other studies; it is an underestimate of the overall repetition rate as those patients who are not admitted after a further parasuicide are not identifiable. ${ }^{212}$ Our estimate of 87000 hospital admissions in England and Wales is slightly lower than Hawton and Fagg's in 1992. However, their estimate of 100000 per year is for hospital referrals (rather than admissions) and is based on figures from Oxford.

There were significant variations in the SARs between districts. These differences are unlikely to be due to coding errors and more probably reflect genuine differences in admission rates. There are two possible explanations for these differences, both of which are likely to contribute to the observed variation. Firstly, differences in socioeconomic status may significantly affect rates of parasuicide. The data show that those districts with higher levels of socioeconomic deprivation, as measured by the Townsend score, tended to have higher parasuicide SARs. A similar ecological link has recently been found for the 24 localities in Bristol. ${ }^{15}$ Using ranks, $9 \%$ of the variation in parasuicide SAR is explained by deprivation. If the outlying district which has the highest parasuicide SAR and admits the greatest proportion of accident and emergency attenders is omitted, $68 \%$ of the variation is explained by deprivation. The other possible explanation for the observed variation is that hospital admission practices for parasuicide vary across the south west.

Further evidence of variations was derived from the postal questionnaire sent to accident and emergency departments in the region. A positive correlation was found between the SAR for parasuicide and the proportion of patients admitted from accident and emergency. The accuracy of the data reported in the postal questionnaire is likely to be variable as the sophistication and completeness of casualty information systems varies across the region (see discrepancies between tables 1 and 2). However, we feel there is no reason to suspect systematic bias in the information we have been given. In addition, for the calculation of the proportion of parasuicide patients admitted we requested information from the departments on both the number of attendees and the number of admissions. Any underestimate or overestimate is likely to affect both the numerator and denominator in this proportion as both were provided by the individual units.

Analysis of the relationship between the management of parasuicide and outcomes is limited by the number of districts. All reported correlations have wide confidence intervals and should be interpreted with caution. Where relationships were found four substantive explanations are possible. Firstly, the data may be incorrect and relationships result from systematic differences in coding practices between hospitals. Secondly, in-patient services may differ between districts - some may have psychiatric units on-site at district general hospitals and this may alter management strategies.

For example, the three districts where the greatest proportion of attendees were admitted had psychiatric inpatient facilities on-site in all their district general hospitals. Thirdly, the severity of casemix may vary between districts and this rather than clinical practice may result in the observed variations in outcome. Lastly, management policies and treatment services for parasuicide are likely to differ; this may result in differences in outcome. The role of each of these factors should be fully explored using more in-depth, focused, and preferably prospective data collection.

About $10 \%$ of admissions are referred for psychiatric inpatient treatment emphasising the importance of parasuicide as a marker for severe psychiatric illness, highlighted by the long term suicide risk. This referral rate is similar to that found in Oxford and Edinburgh. ${ }^{12}$ Surprisingly, our data suggest that there is little relationship between the proportion of patients admitted from accident and emergency and the proportion subsequently referred for psychiatric inpatient care. If some hospitals only admit a population already screened for severity of psychiatric illness in accident and emergency, one would expect such hospitals to have a greater proportion of their patients subsequently admitted for psychiatric care. Our analysis found no evidence to support a policy of admission for all those who self harm. If anything those districts referring a greater percentage of patients for subsequent inpatient psychiatric care experienced a higher rate of repeat parasuicide admission. The basic lack of relationship here may either be because those districts with on-site psychiatric facilities admitted a greater proportion of parasuicide patients or because of local differences in the severity of psychiatric illness among those who deliberately self harm.

A third of suicides have a history of previous parasuicide, and in the year following parasuicide $1 \%$ of individuals succeed in killing 
themselves, with the eventual figure being 3$10 \%{ }^{25-29}$ Mortality statistics show a total of 104 suicide deaths from poisoning in 1992 in the south west, thus about a quarter of these deaths occur in hospital in the South Western Region. Thus at least a quarter of all suicide deaths by overdose in the south west occurred in hospital and it is possible that some of these may be preventable by following best practice management guidelines. Overall, the proportion who died after admission for overdose was $0.5 \%$. These figures underestimate the total number of fatalities among those who reach hospital alive, since some patients with severe liver damage will have been transferred to specialist liver units elsewhere in the country for further management. The figures also underestimate total deaths from overdose as many patients die prior to hospital admission.

The variations seen in the south west have been observed elsewhere and demonstrate uncertainty concerning the best management strategy for these patients. ${ }^{810}$ Such variations may be reduced by consensus statements produced by authoritative bodies. However, as attempts to reduce parasuicide repetition have proved largely unsuccessful ${ }^{16-20}$ except in high risk groups ${ }^{21-23}$ the reasons for variation should be further explored. The effect of different admission policies on outcome should also be examined to clarify best practice guidelines for parasuicide. There remains uncertainty about whether, for most patients who self harm, admission to hospital is beneficial.

Randomised controlled trials are required to determine whether, for those patients where hospital admission is not indicated on medical grounds, admission is beneficial in the management of parasuicide. Management subsequent to acute medical admission also requires further controlled evaluation.

We would like to thank: Professor HG Morgan, Dr J Evans, Dr RM Kammerling, and Mr W White (regional coding officer) for advice, comments and assistance; casualty consultants and managers in the South Western Region and the Information Department Royal United Hospital, Bath for providing us with information on attendances at their departments.

1 Stark C, Smith H, Hall D. Increase in parasuicide in Scotland. BM7 1994;308:1569-70.

2 Hawton K, Fagg J. Trends in deliberate self poisoning and self injury in Oxford, 1976-90. BMF 1992;304:1409-11.

3 Beck P, Lazarus J, Scorer R, Smith P, Routledge P. Increasing rates of deliberate self harm. BMF 1994;308:789.

4 Alderson $M$. National trends in self-poisoning in women. Lancet 1985; :974-5.

5 Secretary of State for Health. The Health of the nation: a strategy for health in England. London: HMSO, 1992.

6 Faculty of Public Health Medicine of the Royal College of Physicians UK Levels of Health. Second Report, July 1992.
London: Faculty of Public Health Medicine 1992;27-29. World Health Organisation. Health for all targets. The health policy for Europe. Copenhagen: WHO, 1993.

8 House A, Owens D, Storer D. Psycho-social intervention following attempted suicide: is there a case for bette services? International Revue of Psychiatry 1992;4:15-22.

9 Department of Health and Social Security. The management of deliberate self harm. Health Notice HN (84)25. London: DHSS, 1984

10 Owens D. Self-harm patients not admitted to hospital. $f$ Roy Coll Phys 1990;24:281-3.

11 Phillimore P, Beattie A, Townsend P. Widening inequality of health in Northern England $B M Y$ 1994;308:1125-1128.

12 Platt S, Hawton K, Kreitman N, Fagg J, Foster J. Recen epidemiological trends in parasuicide in Edinburgh and epidemiological trends in parasuicide in Edinburgh and

13 Cameron I, Renvoize E, Garside G. Deliberate self-poisoning: the need for a new approach. Health Trends 1990 3:126-8.

14 Kennedy P, Kreitman N. An epidemiological survey of parasuicide (attempted suicide) in general practice. $\mathrm{Br} f$ Psychiatry 1973;123:23-34

15 Gunnell DJ, Peters TJ, Kammerling RM, Brooks J. Relation between parasuicide, suicide, psychiatric admissions and socio-economic deprivation. BMF 1995;311:226-30

16 Hawton K, McKeown S, Day A, Martin P, O'Connor M Yule J. Evaluation of out-patient counselling compared with general practitioner care following overdoses. Psychol with general practition $1987 ; 17: 751-61$.

17 Gibbons JS, Butler J, Urwin P, Gibbons JL. Evaluation of social work service for self-poisoning patients. Br f Psych social work service for

18 Chowdhury N, Hicks RC, Kreitman N. Evaluation of an after-care service for parasuicide (attempted suicide) patients. Social Psychiatry 1973;8:67-81.

19 Ettlinger R. Evaluation of suicide prevention after attempted suicide. Acta Psychiatr Scand 1975;260(suppl):1-125.

20 Deykin EY, Hsieh C, Joshi N, McNamarra JJ. Adolescen suicidal and self-destructive behaviour. Results of an intervention study. $\mathcal{F}$ Adolesc Health Care 1986;7:88-95.

21 Linehan MM, Armstrong HE, Suarez A, Allmon D, Heard HL. Cognitive-behavioural treatment of chronically parasuicidal borderline patients. Arch Gen Psychiatry 1991;48: suicidal $1060-4$.

22 Montgomery SA, Montgomery D. Pharmacological prevention of suicidal behaviour. $f$ Affective Disord 1982;4 vention

23 Salkovskis PM, Atha C, Storer D. Cognitive-behavioural Problem solving in the treatment of patients who repeatedly attempt suicide. Br $\mathcal{F}$ Psychiatr 1990;157:871876 .

24 Morgan HG, Jones EM, Owen JH. Royal College of Psychiatrists. The general hospital management of deliberate self harm. A consensus statement on standards for service provision. London: Royal College of Psychiatrists 1994

25 Gunnell D, Frankel S. Prevention of suicide: aspirations and evidence. $B M F$ 1994;308:1227-33.

26 Barraclough B, Bunch J, Nelson B, Sainsbury P. A hundred cases of suicide: clinical aspects. Br $¥$ Psychiatry $1974 ; 125$ : 355-73.

27 Monk M. Epidemiology of suicide. Epidemiol Rev 1987;9. 51-69.

28 Hawton K, Fagg J. Suicide, and other causes of death following attempted suicide. $B r \mathcal{F}$ Psychiatry 1988;152 359-66

29 Nordentoft M, Breum L, Munck L, Nordestgaard AG, Hunding A, Laursen Bjaeldager PA. High mortality by natural and unnatural causes: a 10 year follow up study of patients admitted to a poisoning treatment centre after suicide attempts. BMF 1993;306:1637-41.

30 Hawton K, Fagg J, Simkin S, Mills J. Attempted suicide in Oxford 1991 and 1992. Oxford: University Department of Psychiatry, Warneford Hosptial, and Barnes Unit, 1993.

31 Dennis M, Owens D, Jones S. Epidemiology of deliberate self poisoning: trends in hospital attendences. Health self poisoning: trends
Trends 1990;22:125-6.

32 Sellar C, Goldacre M, Hawton K. Reliability of routine hospital data on poisoning as measures of deliberate self poisoning in adolescents. F Epidemiol Community Health 1990;44:313-15.

33 Breslow NE, Day NE. Statistical methods in cancer research. Vol II. The design and analysis of cohort studies. Lyon:IARC $1987 ; 96$. 